\title{
Intramedullary Spinal Cord Metastases: Prognostic Value of MRI and Clinical Features from a 13-Year Institutional Case Series
}

\author{
F.E. Diehn, J.B. Rykken, J.T. Wald, C.P. Wood, L.J. Eckel, C.H. Hunt, K.M. Schwartz, R.K. Lingineni, R.E. Carter, and T.J. Kaufmann
}

\begin{abstract}
BACKGROUND AND PURPOSE: In patients with intramedullary spinal cord metastases, the impact of MR imaging and clinical characteristics on survival has not been elucidated. Our aim was to identify MR imaging and clinical features with prognostic value among patients with intramedullary spinal cord metastases from a large retrospective series.
\end{abstract}

MATERIALS AND METHODS: The relevant MR imaging examination and baseline clinical data for each patient from a consecutive group of patients with intramedullary spinal cord metastases had previously been reviewed by 2 neuroradiologists. Additional relevant clinical data were extracted. The influence of clinical and imaging characteristics on survival was assessed by Kaplan-Meier survival curves and log-rank tests for categoric characteristics.

RESULTS: Forty-nine patients had 70 intramedullary spinal cord metastases; 10 (20\%) of these patients had multiple metastases. From the date of diagnosis, median survival for all patients was 104 days $(95 \% \mathrm{Cl}, 48-156$ days). One clinical feature was associated with decreased median survival: lung or breast primary malignancy ( 57 days) compared with all other malignancy types ( 308 days; $P<.001)$. Three MR imaging features were associated with decreased median survival: multiple intramedullary spinal cord metastases ( 53 versus 121 days, $P=$ .022), greater longitudinal extent of cord T2 hyperintensity (if $\geq 3$ segments, 111 days; if $\leq 2,184$ days; $P=.018$ ), and ancillary visualization of the primary tumor and/or non-CNS metastases (96 versus 316 days, $P=.012$ ).

CONCLUSIONS: Spinal cord edema spanning multiple segments, the presence of multifocal intramedullary spinal cord metastases, and ancillary evidence for non-CNS metastases and/or the primary tumor are MR imaging features associated with decreased survival and should be specifically sought. Patients with either a lung or breast primary malignancy are expected to have decreased survival compared with other primary tumor types.

ABBREVIATION: ISCM = intramedullary spinal cord metastasis

$\mathbf{R}$ cent studies have elucidated the imaging features of inramedullary spinal cord metastases (ISCMs). MR imaging findings specific to these secondary compared with primary tumors of the spinal cord have been described. ${ }^{1}$ Moreover, multiple MR imaging characteristics have been characterized in detail $^{2}$ and correlated with findings on physiologic imaging with PET. ${ }^{3}$ However, the prognostic value of MR imaging and clinical findings has not been assessed, to our knowledge. A recent literature review of

\footnotetext{
Received June 16, 2014; accepted after revision September 6.

From the Division of Neuroradiology (F.E.D., J.B.R., J.T.W., C.P.W., L.J.E., C.H.H., K.M.S., T.J.K.), Department of Radiology, and Department of Health Sciences Research (R.K.L., R.E.C.), Mayo Clinic, Rochester, Minnesota.

Paper previously presented at: American Society of Neuroradiology Annual Meeting and the Foundation of the ASNR Symposium, May 17-22, 2014; Montreal, Quebec, Canada.

Please address correspondence to Felix E. Diehn, MD, Department of Radiology, Mayo Clinic, 200 First St SW, Rochester, MN 55905; e-mail: diehn.felix@mayo.edu

http://dx.doi.org/10.3174/ajnr.A4160
}

301 patients noted that there are no evidence-based treatment guidelines for ISCMs and that the various therapeutic options do not generally considerably affect survival. ${ }^{4}$ Thus, identification of pretreatment factors that may affect outcomes is relevant. The purpose of this retrospective study was to identify MR imaging and clinical features with prognostic value among patients with ISCMs from a large retrospective series.

\section{MATERIALS AND METHODS}

Institutional review board approval with waived consent was obtained for this Health Insurance Portability and Accountability Act-compliant retrospective research study.

\section{Subjects}

As detailed in a prior study, ${ }^{2}$ the pathologic, surgical, clinical, and radiologic data bases at our single institution were queried to identify a group of consecutive patients with ISCMs $(n=58$, 1999-2011). Exclusion criteria were patients in whom ISCM was 
never formally clinically diagnosed, patients in whom alternative diagnoses were suspected clinically, and patients without available pretreatment digital MR images. This group of patients with ISCMs is from the same 58 patients in whom we recently described 2 specific enhancement features of ISCM on postgadolinium MR imaging that may help to differentiate it from primary cord masses. ${ }^{1}$ Five (9\%) of these 58 patients were again excluded due to a lack of available pretreatment MR imaging examinations. Additional details of the study population were as reported in the prior studies ${ }^{1,2}$; specifically, 4 (8\%) of the remaining 53 patients "were excluded because alternative diagnoses were being considered clinically and ISCM was never formally diagnosed." ${ }^{1}$ Thus, only patients with a clinical diagnosis of ISCM were included on the basis of review of available clinical, pathologic, and imaging data. The resultant final ISCM study population was 49 with 70 ISCMs, with solitary ISCMs in 39 and multiple in 10. In 5 (10\%) of these 49 patients, the diagnosis of ISCM was confirmed by cord mass biopsy/resection. In 44 (90\%) of the 49 patients with ISCM, "pathologic proof of the systemic malignancy had been obtained from the primary site or a metastatic site outside of the spinal cord." 1,2

\section{MR Imaging Review}

Two radiologists had previously reviewed all MR imaging examinations in consensus at an electronic workstation (one [F.E.D.], a staff neuroradiologist with American Board of Radiology certification and a Certificate of Added Qualification in neuroradiology, and the other [J.B.R.], a neuroradiology fellow with American Board of Radiology certification). ${ }^{2}$ The MR imaging examinations were predominantly from our institution, but some were from outside facilities. Given this range of institutions and the $>10$-year timeframe of this retrospective study, no single, consistent MR imaging platform or protocol was used. At our institution, a typical MR imaging protocol for a patient suspected of having a spinal cord neoplasm during the timeframe of the study consisted of the following sequences, at 1.5T field strength: precontrast sagittal T1WI, T2WI, and optional STIR optimized for spinal cord signal abnormality (TI $\sim 110 \mathrm{~ms}$ ); axial T2WI; optional axial gradient recalled-echo and axial T1WI; and postcontrast sagittal and axial T1WI.

The results of this detailed radiologic review have been published in a separate study. ${ }^{2}$ Previously analyzed radiologic features that are relevant for the present study are the following: number of ISCMs per patient, and for each ISCM: 1) maximal size of the enhancing lesion in millimeters (measured anteroposterior, transverse [axial images required], and superoinferior), 2) maximal longitudinal extent of the enhancing lesion in a number of vertebral segments, 3) maximal longitudinal extent of spinal cord $\mathrm{T} 2$ hyperintensity in a number of vertebral segments (sagittal T2 images required), 4) position within the cord on axial images (central, eccentric, exophytic), 5) cord expansion (absent versus present), 6) T2 signal intensity (hyper-, hypo-, or isointense to spinal cord), 7) T1 signal intensity (hyper-, hypo-, or isointense to spinal cord), 8) convincing evidence of cystic change (absence or presence of nonenhancing fluid signal on T1- and T2-weighted images), 9) convincing evidence of intratumoral hemorrhage (absence or presence of nonenhancing T1 hyperintensity and/or marked T1 hypointensity and corresponding T2 hypointensity and/or "blooming" magnetic susceptibility artifacts on gradient recalled-echo sequences, if available), and 10) gadolinium enhancement pattern (absent, or if present, homogeneous versus heterogeneous versus ring). ${ }^{2}$ Presence or absence of postgadolinium features highly specific for ISCM ("rim" and "flame" signs) was also based on previously obtained data. ${ }^{1}$ As per that prior report, the definitions of these signs are the following: rim sign, "a complete or partial thin peripheral rim of gadolinium enhancement more intense than the central enhancement of a noncystic/necrotic lesion"; flame sign, "an ill-defined flameshaped region of gadolinium enhancement at the superior and/or inferior margin of an otherwise well-defined lesion"; and "both the rim and flame signs can be present in the same lesion." ${ }^{11}$ The ratio of the length of spinal cord T2 hyperintensity to the length of the ISCM was calculated for each lesion.

For each patient, the reference spinal MR imaging had previously been reviewed for ancillary evidence of the primary tumor and/or extraspinal, non-CNS metastases and any other spinal column and/or CNS metastases not involving the spinal column (including vertebral column, leptomeninges, and visualized intracranial contents). ${ }^{2}$ When available, follow-up spinal MR imaging examinations had also been reviewed for the development of additional ISCMs. ${ }^{2}$ A previous study had categorized the presence or absence of uptake of these ISCMs on PET, when this was available, within 60 days prior or 14 days after the correlative pretreatment diagnostic MR imaging. ${ }^{3}$

Note that a previously published analysis ${ }^{2}$ of the 70 ISCMs in the current study demonstrated that most of the lesions had a convincing appearance of being intramedullary rather than leptomeningeal in origin. Specifically, only 4 of the lesions were exophytic on MR imaging, and in all 4 of these cases, the interpreting radiologist had described an intramedullary mass extending exophytically, rather than a leptomeningeal mass invading the parenchyma. Only 3 lesions ( $4 \%$ of 70 ISCMs) appeared to be potentially leptomeningeal in origin, with 2 occurring in the same patient. ${ }^{2}$

\section{Clinical Review}

The electronic medical records of each patient with ISCM were reviewed. The primary tumor type and the dates of the primary tumor diagnosis, ISCM clinical presentation, and ISCM diagnosis had previously been noted. ${ }^{2}$ The date of death (if applicable) was identified for each patient. We calculated 2 relevant intervals: survival from ISCM clinical presentation and survival from ISCM diagnosis.

\section{Statistical Analysis}

The statistical evaluation consisted of performing analyses on all subjects (having either solitary or multiple ISCMs) and on subjects with only solitary ISCMs. Median survival (days, [95\% CI]) from the date of the ISCM diagnosis was calculated for each of the following variables by using the Kaplan-Meier product limit estimator for the following: 1) clinical variables: age, primary malignancy type, lung/breast versus nonlung/nonbreast primary malignancy, melanoma versus nonmelanoma primary malignancy, CNS versus non-CNS primary malignancy, and ISCM diagnosis preceding primary malignancy diagnosis; and 2) MR imaging variables: solitary versus multifocal ISCMs, non-spinal cord CNS 
Table 1: Survival analysis of clinical features for all patients with ISCM (N=49)

\begin{tabular}{|c|c|c|c|}
\hline Variable & $\begin{array}{l}\text { Median Survival } \\
\left(\text { days }^{\mathrm{a}}\right)(95 \% \mathrm{Cl})\end{array}$ & $\begin{array}{l}\text { Hazard Ratio } \\
(95 \% \mathrm{Cl})\end{array}$ & $P$ Value \\
\hline Age at diagnosis $(\mathrm{yr})(\mathrm{N}=49)$ & $108(48-156)$ & $1.02(0.99-1.04)$ & .091 \\
\hline Primary malignancy & & & .005 \\
\hline Lung $(n=24)$ & $58(31-120)$ & - & \\
\hline Breast $(n=7)$ & $48(21-456)$ & - & \\
\hline Melanoma $(n=5)$ & $87(15-734)$ & - & \\
\hline CNS $(n=4)$ & 763 (19-1261) & - & \\
\hline Renal $(n=3)$ & $299(231-643)$ & - & \\
\hline Other $(n=6)$ & $503(24-884)$ & - & \\
\hline Lung/breast primary malignancy & & & $<.001$ \\
\hline Nonlung/nonbreast $(n=18)$ & 308 (87-689) & - & \\
\hline Lung/breast ( $n=31$ ) & $57(33-117)$ & - & \\
\hline Melanoma & & & .635 \\
\hline Nonmelanoma $(n=44)$ & $115(40-166)$ & - & \\
\hline Melanoma $(n=5)$ & $87(15-734)$ & - & \\
\hline CNS primary malignancy & & & .018 \\
\hline Non-CNS $(n=45)$ & $104(42-127)$ & - & \\
\hline CNS $(n=4)$ & 763 (19-1261) & - & \\
\hline $\begin{array}{l}\text { ISCM diagnosis precedes primary } \\
\text { malignancy diagnosis }\end{array}$ & & & .361 \\
\hline Noo $(n=44)$ & $104(42-184)$ & - & \\
\hline Yes $(n=5)$ & $127(21-187)$ & - & \\
\hline
\end{tabular}

Note:- - indicates not applicable.

a From the date of ISCM diagnosis.

${ }^{b} P$ values are for log-rank tests for categoric variables and Wald tests for continuous variables.

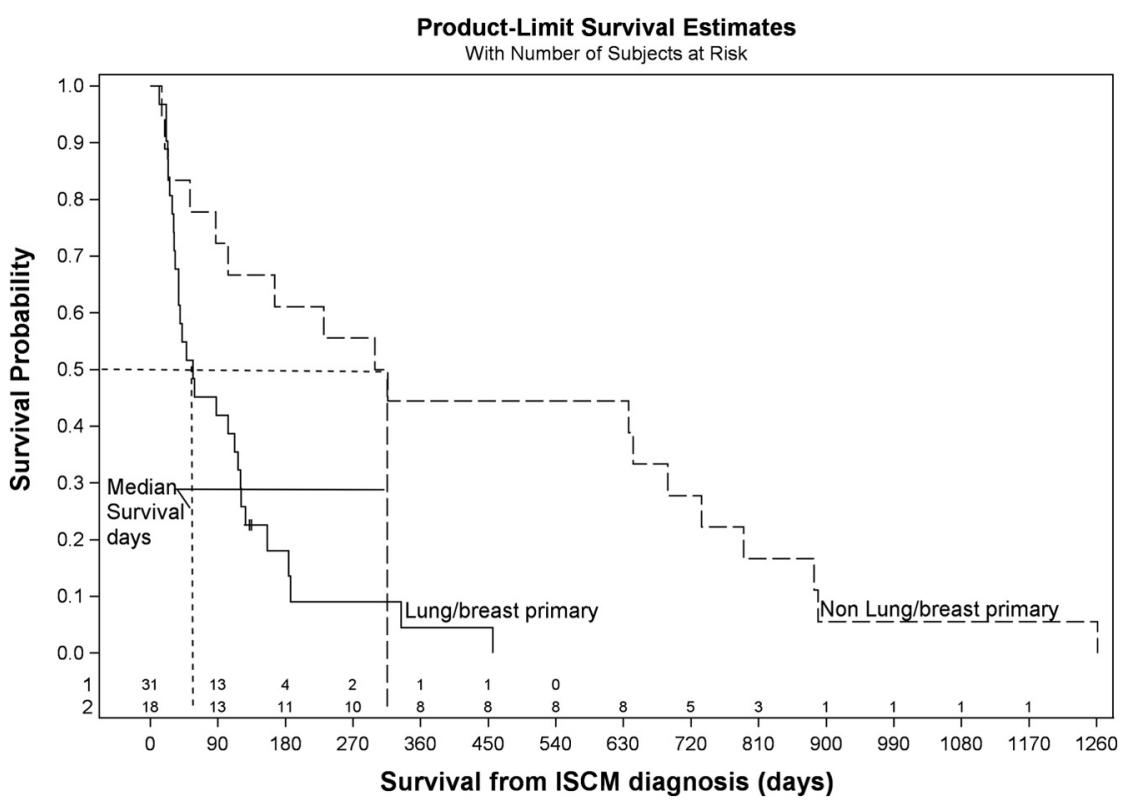

Lung/Breast primary $\quad$ 1: Lung/Breast $\quad---\cdot 2$ : Non Lung/Breast

FIG 1. Kaplan-Meier plot. Decreased survival for patients with lung or breast primary malignancy compared with all other types of primary malignancy. The median survival for patients with lung or breast primaries was 57 days and for patients with nonlung/nonbreast primaries was 308 days $(P<.001$, log-rank test; $N=49$ patients).

and/or spinal metastases, leptomeningeal metastases, primary tumor and/or non-CNS metastases, longitudinal extent of spinal cord T2 hyperintensity (treated both as continuous and categoric [ $\geq 2$ and $\geq 3$ segments] variables), ISCM enhancement size (millimeters, superoinferior), ISCM enhancement longitudinal extent (number of segments), ratio of the extent of longitudinal cord T2 hyperintensity to longitudinal ISCM enhancement, and presence/ absence of rim and flame signs (alone or in combination).

The rationale for analyzing lung/breast primary malignancy compared with others was that the 10 cases of multiple ISCMs in this patient population occurred only with these 2 malignancies. ${ }^{2}$ In addition, this grouping of lung and breast primary malignancies was driven by both the relatively small number of patients with breast primary malignancy $(n=7)$, and the apparent clustering of the shorter survival curves for these 2 primary malignancies and clustering of longer survival curves of the other primary malignancies at initial statistical analysis. The rationale for analyzing melanoma primary malignancy compared with others was that prior studies have suggested improved survival in patients with melanoma., The rationale for using the date of ISCM diagnosis rather than the date of ISCM presentation was the following: 1) The former was deemed the more relevant time index from a patient care perspective and has been used in other studies such as the recent comprehensive literature review by Sung et $\mathrm{al}^{4} ; 2$ ) there was a median survival difference of only 4 days by using these 2 different time indices; and 3) although the granular data are not included herein, the results were comparable between the 2 dates. Logrank tests and large sample tests of the hazard ratios (Wald tests) were used, respectively, to compare the potential effect on survival of each of these categoric and continuous variables. The hazard ratios were estimated from a Cox model. For the T2 signal abnormality and ISCM enhancement characteristics in patients with multiple ISCMs, the length of the longest lesion was used, and the rim and flame signs were considered to be positive if present in any 1 of the multiple ISCMs. Patients who were still alive or lost to follow-up were censored at the last known date alive for the analysis.

Statistical analyses were conducted by using SAS, Version 9.3 (SAS Institute, Cary, North Carolina). Statistical significance was defined by $P<.05$.

\section{RESULTS}

\section{Survival}

The date of death was known in 96\% (47/49) of patients; $4 \%$ of patients $(2 / 49)$ were either still alive $(n=1)$ or lost to follow-up $(n=1)$ by the end of the analysis period. From the dates of ISCM diagnosis and presentation, median survival for all patients was 104 days (3.5 months; 95\% CI, 48-156 days) and 108 days (3.6 months; 95\% CI, 77-166 days), respectively. 
Table 2: Survival analysis of MRI features for patients with solitary ISCMs ( $n=39$ )

\begin{tabular}{|c|c|c|c|}
\hline Variable & $\begin{array}{l}\text { Median Survival } \\
\left(\text { days }^{\mathrm{a}}\right)(95 \% \mathrm{Cl})\end{array}$ & $\begin{array}{l}\text { Hazard Ratio } \\
(95 \% \mathrm{Cl})\end{array}$ & $P$ Value $^{\mathrm{b}}$ \\
\hline No. of ISCMs & & & .022 \\
\hline Solitary $(n=39)$ & $121(42-187)$ & - & \\
\hline Multiple $(n=10)$ & $53(12-88)$ & - & \\
\hline Noncord CNS/spinal metastases & & & .879 \\
\hline No $(n=19)$ & $166(87-334)$ & - & \\
\hline Yes $(n=20)$ & $79(26-184)$ & - & \\
\hline Leptomeningeal metastases & & & .274 \\
\hline No $(n=27)$ & $127(38-231)$ & - & \\
\hline Yes $(n=12)$ & $113(24-790)$ & - & \\
\hline Primary tumor/non-CNS metastases & & & .012 \\
\hline No $(n=15)$ & $316(38-790)$ & - & \\
\hline Yes $(n=24)$ & $96(32-166)$ & - & \\
\hline Cord $\mathrm{T} 2$ hyperintensity $\geq 2$ segments $^{\mathrm{c}}$ & & & .037 \\
\hline No $(n=10)$ & $184(24-884)$ & - & \\
\hline Yes $(n=28)$ & $111(38-166)$ & - & \\
\hline Cord $\mathrm{T} 2$ hyperintensity $\geq 3$ segments ${ }^{\mathrm{c}}$ & & & .018 \\
\hline No $(n=12)$ & $184(24-790)$ & - & \\
\hline Yes $(n=26)$ & $111(38-166)$ & - & \\
\hline Cord T2 hyperintensity (No. of segments) ${ }^{c}(n=38)$ & $121(42-187)$ & $1.02(0.96-1.09)$ & .541 \\
\hline ISCM enhancement, superoinferior size $(\mathrm{mm})^{c}(n=35)$ & $120(42-187)$ & $1.01(0.99-1.02)$ & .138 \\
\hline $\begin{array}{l}\text { ISCM enhancement, extent (No. of vertebral segments) }{ }^{c} \\
\quad(n=35)\end{array}$ & $120(42-187)$ & $1.08(0.85-1.38)$ & .524 \\
\hline $\begin{array}{l}\text { Ratio, longitudinal extent of cord T2 hyperintensity to } \\
\text { enhancement }{ }^{\mathrm{C}}(n=34)\end{array}$ & $119(40-187)$ & $1.03(0.94-1.13)$ & .477 \\
\hline $\operatorname{Rim} \operatorname{sign}^{c}$ & & & 621 \\
\hline No $(n=18)$ & $79(26-643)$ & - & \\
\hline Yes $(n=17)$ & $127(40-299)$ & - & \\
\hline Flame sign ${ }^{c}$ & & & .068 \\
\hline No $(n=19)$ & $127(42-734)$ & - & \\
\hline Yes $(n=16)$ & $96(26-299)$ & - & \\
\hline Rim/flame signs ${ }^{c}$ & & & .143 \\
\hline Neither $(n=13)$ & $121(32-884)$ & - & \\
\hline Rim sign only $(n=6)$ & $147(21-790)$ & - & \\
\hline Flame sign only $(n=5)$ & $26(15-643)$ & - & \\
\hline Both signs $(n=11)$ & $120(38-316)$ & - & \\
\hline
\end{tabular}

Note:- - indicates not applicable.

${ }^{a}$ From date of ISCM diagnosis.

${ }^{\mathrm{b}} P$ values are for log-rank tests for categoric variables and Wald tests for continuous variables.

'Missing patients $(n<39)$ due to MRI not having sagittal T2- or gadolinium-enhanced sagittal T1-weighted images.

\section{Prognostic Value of Clinical Features}

Table 1 demonstrates the impact of various clinical features on survival from the date of ISCM diagnosis, considering all 49 patients. The median survival of patients with ISCMs with lung or breast primary malignancies (1.9 months) was shorter than that of patients with all other types of primary malignancy (10.3 months $)(P<.001)$. This association was maintained when the analysis was performed on only the 39 patients with solitary ISCMs $(P=.002$, with respective median survival of 3.5 months for lung/breast primaries [ $n=21]$ and 10.3 months for nonlung, nonbreast primaries $[n=18])$. A Kaplan-Meier plot for all patients with ISCMs demonstrates the shorter survival period for patients with lung/breast primary malignancies (Fig 1). When analyzed by each primary malignancy individually, the different distribution, with both lung and breast each having shorter survival, maintained statistical significance, though most of the individual primary types had a small sample size (Table $1, P=.005$ ). This statistical significance was also maintained when the analysis was performed on only the 39 patients with solitary ISCMs $(P=$ .017 , with respective median survival of 3.9 months for lung primaries [ $n=17]$ and 1.3 months for breast primaries $[n=4]$ ). Additional median survivals based on primary malignancy considering both solitary and multiple ISCMs were the following: lung (1.9 months, $n=24)$, breast (1.6 month, $n=7)$, melanoma (2.9 months, $n=5)$, all nonmelanoma primaries (3.8 months, $n=44)$, CNS (25.4 months, $n=4)$, renal (10.0 months, $n=3)$, and other (16.8 months, $n=6)$. The median survival of patients with ISCMs with non-CNS primary malignancies (3.5 months) was shorter than that of patients with CNS primary malignancy (25.4 months) $(P=.018)$, though the sample size of CNS primaries was small. None of the following features significantly correlated with survival: age at ISCM diagnosis (mean and median, 58 years), melanoma-versus-nonmelanoma primary malignancy, and ISCM diagnosis preceding primary malignancy diagnosis.

\section{Prognostic Value of MR Imaging Features}

Patients with multiple ISCMs experienced shorter median survivals from the date of ISCM diagnosis than those with solitary ISCMs (1.8 versus 4.0 months, respectively; $P=.022$ ) (Table 2 and Fig 2). Table 2 also demonstrates the impact of various additional categoric and continuous MR imaging findings on patient survival, considering only the 39 patients with solitary ISCMs. Two other MR imaging features were associated with statistically significant shorter survival periods: 1$)$ greater longitudinal extent of T2 signal abnormality $(P=.037$ if at least 2 segments, $P=$ .018 if at least 3 segments) (Fig 3 ); and 2 ) ancillary presence of the primary tumor and/or non-CNS metastases on the reference MR imaging $(P=.012)$ (Fig 4$)$. The correlation between these $2 \mathrm{MR}$ imaging features and a shorter survival period was maintained when the analysis was performed on all 49 patients with either solitary or multiple ISCMs (respective $P$ values for T2 signal abnormality on at least 2 segments, .042; T2 signal abnormality on at least 3 segments, .022; and primary tumor/non-CNS metastases, .012 ). Regarding the longitudinal extent of the T2 signal abnormality, patients with at least 3 segments of spinal cord T2 hyperintensity associated with the solitary ISCM had median and mean survival durations of 111 days (3.7 months) and 157 days (5.2 months), compared with 184 days (6.1 months) and 436 days (14.5 months), respectively, in patients with $\leq 2$ segments of cord T2 hyperintensity. A Kaplan-Meier plot for all patients with solitary ISCMs demonstrated the shorter survival associated with $\geq 3$ segments of cord T2 hyperintensity (Fig 5). When treated as a continuous rather than categoric variable, the number of segments of T2 signal abnormality did not significantly correlate with survival (hazard ratio, 1.02; 95\% CI, 0.96-1.09; $P=.541$ ).

In patients with solitary ISCMs, the presence of a flame sign on 


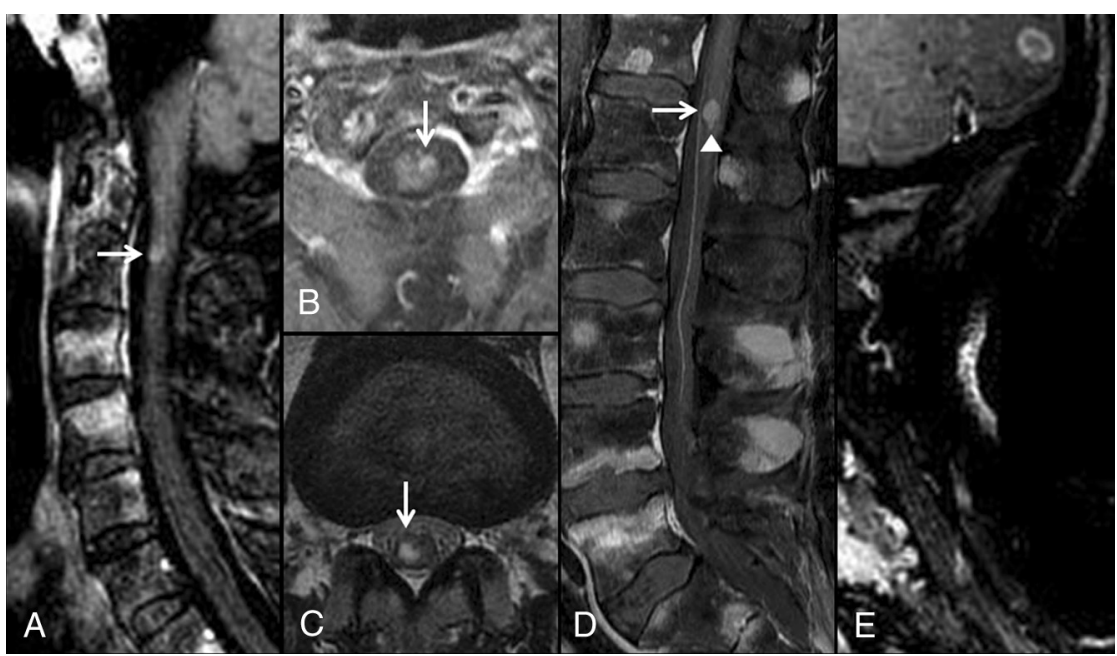

FIG 2. The presence of multiple ISCMs was a poor prognostic indicator. A 76-year-old man with metastatic non-small cell lung cancer. Lung or breast carcinoma was a poor prognostic feature compared with all other primary malignancy types. MR images of the cervical and lumbar spine with postcontrast fat-saturated sagittal $(A, D$, and $E)$ and axial $(B)$ T1-weighted and axial T2weighted $(C)$ images are shown. ISCMs are present at C2 (arrows, $A$ and $B$ ) and L1 (arrows, C and D). Compared with solitary ISCMs, multiple ISCMs were associated with decreased survival. This patient survived 59 days from the diagnosis of ISCMs. The median survival of patients with multiple ISCMs was 53 days, compared with 121 days for those with a solitary ISCM. The conus lesion demonstrates the flame sign inferiorly (arrowhead, D); the presence of this sign was associated with a non-statistically significant trend toward shorter survival. Note that multiple enhancing noncord CNS and spinal (osseous) metastases are present, including in the cerebellum $(E)$; their presence did not correlate with a shorter survival.

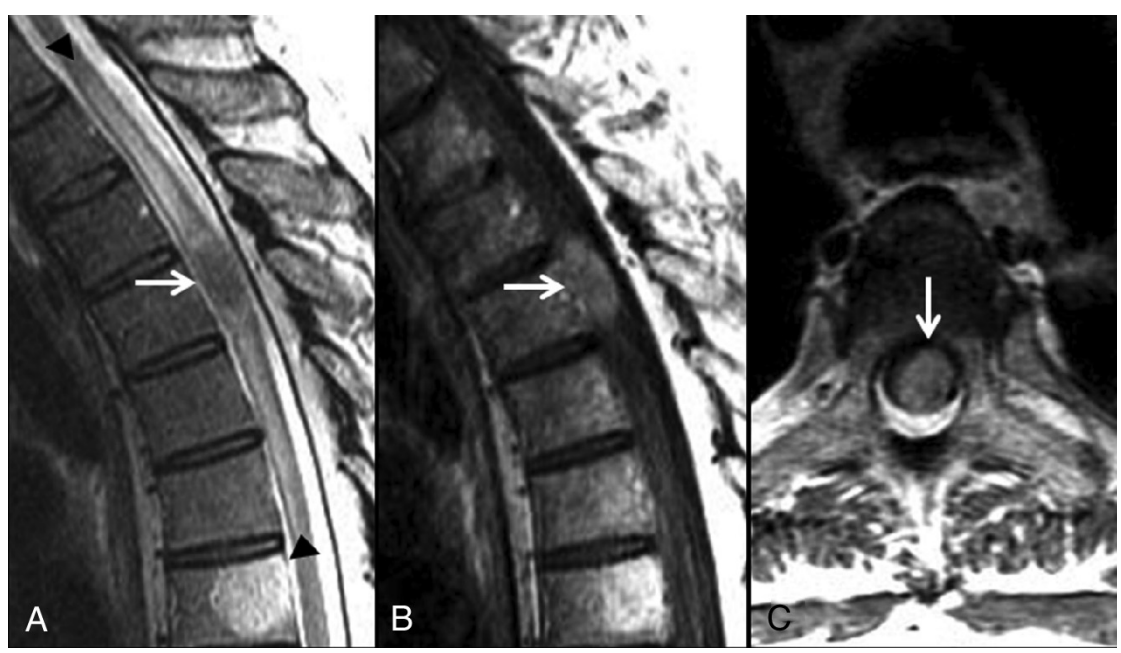

FIG 3. The presence of extensive edema was a poor prognostic indicator. A 55 -year-old man with metastatic cerebral glioblastoma. MR images of the thoracic spine with sagittal T2- $(A)$ and postcontrast T1-weighted sagittal $(B)$ and axial $(C)$ images are shown. An ISCM is present at T4 (arrows, $A-C$ ). Extensive edema for lesion size spans 5 vertebral segments, from the T1-T2 to T6-T7 levels (arrowheads, A). Spinal cord edema spanning multiple segments was associated with decreased survival in this series. This patient survived 19 days from the diagnosis of ISCMs. The median survival of patients with cord edema spanning $\geq 3$ segments was 111 days, compared with 184 days for patients in whom it spanned $\leq 2$ (note that the $T 7$ vertebral body lesion is an incidental hemangioma).

postgadolinium T1-weighted imaging ${ }^{1}$ may be associated with shorter survival; this possible association approached but did not reach statistical significance $(P=.068)$. The following MR imaging findings were not predictive of survival: noncord CNS and/or spinal metastases $(P=.879)$, the presence of leptomeningeal metastases $(P=.274)$, size or extent of ISCM enhancement $(P=$ .138; $P=.524)$, ratio of cord $\mathrm{T} 2$ hyperintensity to enhancement $(P=.477)$, or the rim sign $(P=.621) .{ }^{1}$ Several additional imaging features not shown in Table 2 did not correlate with survival, when considering the 39 patients with solitary ISCMs: position within the cord $(P=.854)$, cord expansion $(P=.169)$, T2 signal intensity $(P=.090)$, T1 signal intensity $(P=.467)$, cystic change $(P=.583)$, intratumoral hemorrhage $(P=.601)$, and gadolinium enhancement pattern $(P=$ .326). In the small group of patients with solitary ISCMs who had available PET examinations $(n=9)$, uptake on PET $(n=6)$ did not correlate with survival $(P=.702)$.

The visualization of additional ISCMs on follow-up MR imaging occurred in 11 of $49(22 \%)$ patients, 10 of whom initially had solitary ISCMs. When we considered all 49 patients, this feature was associated with longer survival: $P=.001$, with respective median survival of 21.2 months for patients in whom this did occur $(n=$ 11) and 1.9 months for patients in whom it did not $(n=38)$. This association was maintained when the analysis was performed on only the 39 patients with solitary ISCMs $(P=.007$, with respective median survival of 21.2 months $[n=10]$ and 3.5 months $[n=29])$.

\section{DISCUSSION}

The current study highlights that in a large series of patients, several clinical and MRI features of ISCMs have prognostic value. Patients with lung or breast primary tumor type have decreased survival compared with patients with all other primary malignancies, a finding that was evident when these primary malignancies were analyzed both as grouped and individual variables. On reference MR imaging, features associated with decreased survival are the following: 1) multiple ISCMs, 2) longitudinally extensive spinal cord T2 hyperintensity, and 3) ancillary presence of the primary tumor and/or non-CNS metastases.

Familiarity with these prognostically relevant clinical and imaging features of ISCMs is relevant to both radiologists and the referring clinicians. For any spinal cord mass, but particularly when ISCM is strongly considered, MR imaging should be scrutinized for the ancillary presence of the primary tumor/non-CNS metastases to assist with the differential diagnosis and evaluate evidence of widely metastatic disease. The visualized lungs should be specifically carefully evaluated on MR imaging because lung cancer is the most com- 


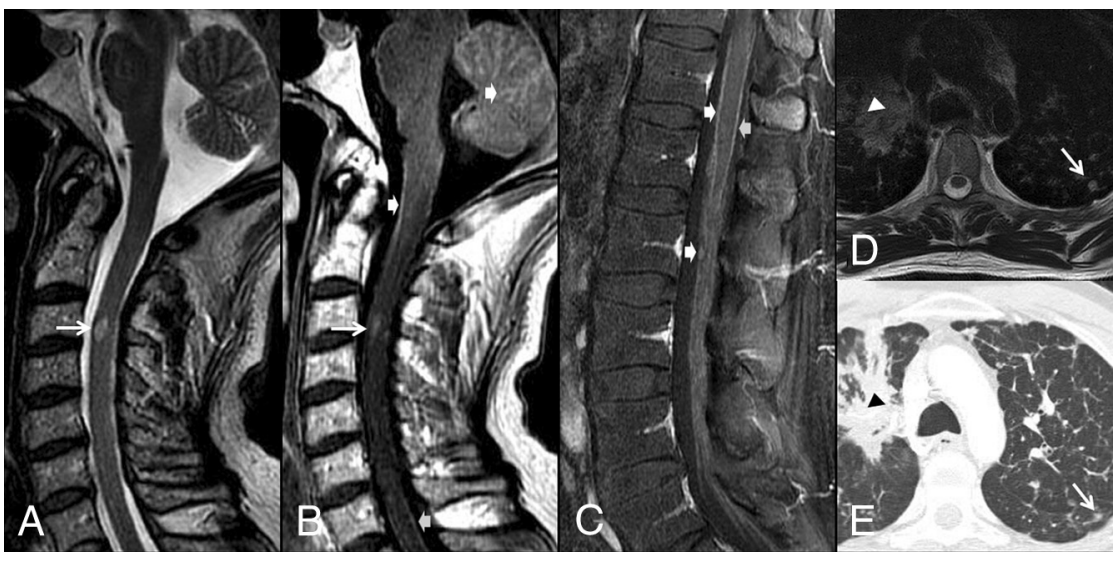

FIG 4. The ancillary presence of primary malignancy and/or non-CNS metastases on reference MR imaging was a poor prognostic indicator. A 39-year-old man with metastatic non-small cell lung cancer. MR images of the cervical, thoracic, and lumbar spine with sagittal T2- (A), postcontrast T1-weighted $(B)$, fat-saturated T1-weighted $(C)$, and axial T2-weighted $(D)$ images, and an axial postcontrast chest CT image (E) are shown. An ISCM is present at C3 (arrows, $A$ and B). The primary lung carcinoma is visualized in the right upper lobe (arrowheads, $D$ and $E$ ), as are several presumed pulmonary metastases (eg, arrows, $D$ and $E$ ). The presence of the primary malignancy and/or non-CNS metastases was associated with decreased survival in this series. This patient survived 24 days from the diagnosis of ISCM. The median survival of patients with visible primary malignancy/non-CNS metastases was 96 days, compared with 316 days for patients in whom these were not visible. Note that leptomeningeal metastases are present (block arrows, $B$ and $C$ ); their presence did not correlate with a shorter survival.

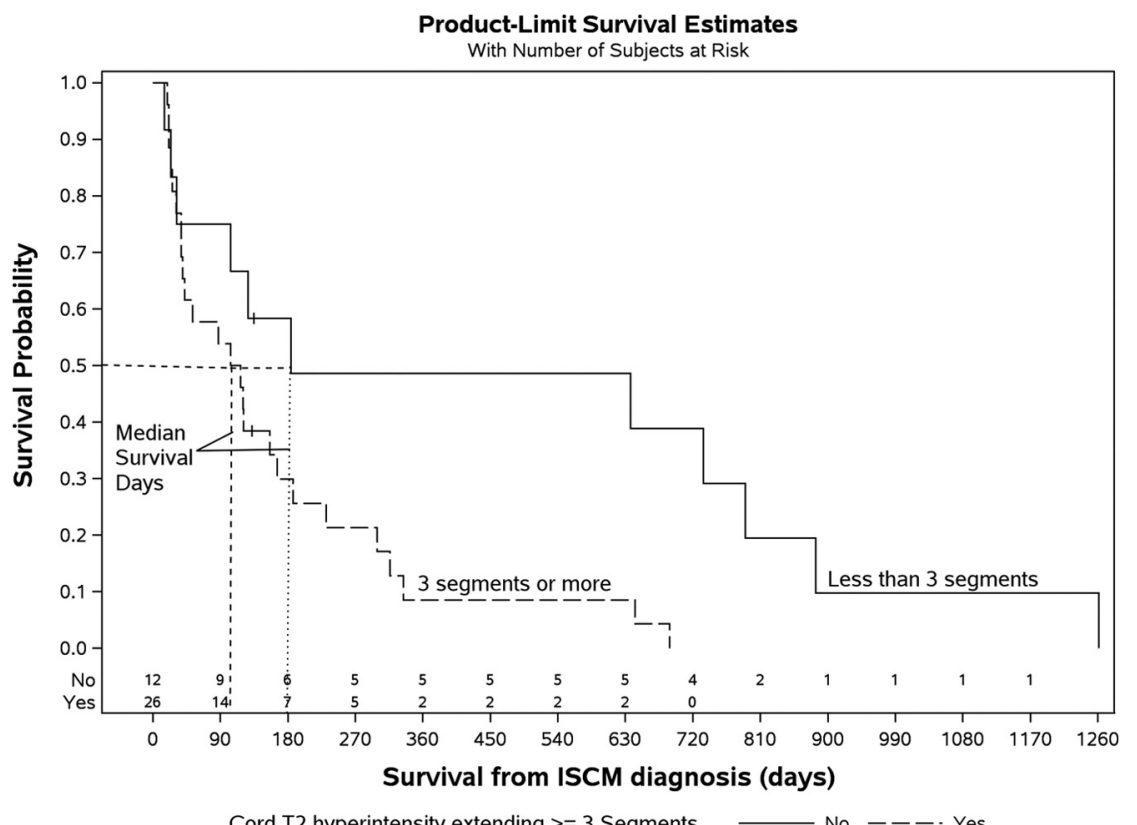

FIG 5. Kaplan-Meier plot. Decreased survival of patients with ISCM-related cord T2 hyperintensity extending $\geq 3$ spinal segments. The median survival for $T 2$ hyperintensity of $\geq 3$ segments was 111 days and for $<3$ segments was 184 days $(P=.018$, log-rank test; $n=38$ patients with solitary ISCMs with available sagittal T2WI).

mon primary malignancy and imparts a worse prognosis compared with other nonlung/nonbreast malignancies. The presence of both extensive spinal cord T2 hyperintensity and additional ISCMs should be specifically sought. Evaluating these MR imaging features not only assists with the differential diagnosis but also offers prognostic value, because patients whose MR imaging examinations have these features have decreased survival.
The decreased survival of those patients with MR imaging evidence of multiple ISCMs and those with the ancillary presence of the primary tumor and/or non-CNS metastases on the reference MR imaging, while intuitive, has not, to our knowledge, been reported in the literature. Although not specifically evaluated in our study, presumed causes for the decreased survival are that these findings represent a particularly aggressive type of advanced (stage IV) metastatic disease, recalcitrance of the disease (either the primary and or the metastases) to therapy, and/or in select cases, the tendency for clinicians and their patients to choose palliative therapy. Likely a complex interplay of factors such as inherent disease aggressiveness, responsiveness to treatment, patient comorbidities, and patient immune system status underlies a patient's ability to have prolonged survival, but the current study, by its very design, cannot shed discriminatory light on these features. It would be interesting to explore these in future studies. The decreased survival associated with greater spinal cord edema extent has also, to our knowledge, not been described, and precise conclusions cannot be drawn about the reason for this association. Presumably such a finding either directly impairs the ability of the patient to survive (eg, perhaps spinal cord functional impairment, possibly due to a more locally infiltrative phenotype) and/or it serves as a marker for a more aggressive disease state (eg, perhaps disease less likely to respond to therapy and/or aggressive metastatic disease to sites outside the spinal cord).

We cautiously interpret the apparent decreased survival of patients with non-CNS versus CNS primary neoplasms as merely an observed possible association, given the very small $(n=4)$ number of patients in the CNS primary neoplasm group and the several CNS histologies that are represented (as previously reported, ${ }^{1} 2$ medulloblastomas, 1 esthesioneuroblastoma, and 1 cerebral glioblastoma). Similarly, the apparent association of visualization of subsequent ISCMs with improved survival must be carefully interpreted. At least in part, this should simply reflect the longer survival of these patients who are being imaged again, allowing documentation of additional ISCM development. Potentially, having imaging of the initial ISCM at an earlier disease state could also contribute to this presumed statistical artifact. Most interest- 
ing, the presence of leptomeningeal disease on MR imaging was not associated with shorter survival. While speculative, a potential explanation is that perhaps such metastatic disease might not impair vital functions as much as a metastasis elsewhere, such as in the spinal cord parenchyma or in other vital organs. The lack of correlation of ISCM diagnosis preceding primary malignancy diagnosis with shorter survival is also interesting. However, even if such a statistical association were present, it would likely be difficult to ascribe significance because of the very small sample size of 5 patients.

The lack of a convincing association of the recently described rim and flame signs ${ }^{1}$ with survival in the current study has yet to be reported. Likewise, the decreased survival of patients with ISCMs with lung or breast primary tumors has not been specifically described. Note that 2 studies previously reported that patients with melanoma had significantly longer survival compared to patients with nonmelanoma histology, ${ }^{5,6}$ a finding not corroborated by our present data, which included 5 patients with melanoma. The latter of the 2 studies based this assertion on the combination of a single patient with melanoma combined with 4 others from the literature, while the former included 2 patients with melanoma. Similarly, the reported longer survival of patients with breast cancer compared with those with lung cancer described in a prior study of 12 patients with ISCMs ${ }^{7}$ was not confirmed in the present study. A similarity between our series and the recent comprehensive literature review by Sung et $\mathrm{al}^{4}$ is the short survival of patients with ISCMs (median, 3.5 months versus 4 months in that review).

Limitations include the retrospective nature of our study. Despite this group being the largest published single institution series of patients with ISCM to our knowledge, the 49-patient sample size is, nonetheless, a limitation. Similarly, the lack of a standard MR imaging technique, given that the examinations were performed during more than a decade and at $>1$ institution, is a weakness. Admittedly, visualization of the primary tumor on spinal MR imaging is not possible in all cases of ISCM due to the area of interest being either not well-included or not being included at all. Multiple possible confounding variables could influence the survival analyses and were not considered, such as extent of metastatic disease clinically at the time of reference MR imaging, comorbidities, and treatment. Because the purpose was to assess the prognostic value of baseline clinical and MR imaging findings irrespective of subsequent therapies, this study did not assess the impact on survival of the various treatment modalities for ISCM or how treatment may or may not have been affected by clinicians' knowledge of the existence of ISCMs. Interested readers are referred to the aforementioned recent comprehensive literature review by Sung et $\mathrm{al}^{4}$; note that even in that review, median survival between surgically and conservatively treated patients was not considerably different (6 versus 5 months, respectively, with solitary-versus-multiple ISCM status not provided).

\section{CONCLUSIONS}

We describe the prognostic value of pertinent clinical and MR imaging features in a large series of patients with ISCMs. Spinal cord edema spanning multiple segments, the presence of multifocal ISCMs, and ancillary evidence for non-CNS metastases and/or the primary tumor should be specifically sought because these MR imaging features are associated with decreased survival. Patients with either a lung or breast primary malignancy have decreased survival compared with other primary tumor types.

Disclosures: Timothy J. Kaufmann—UNRELATED: Consultancy: SpineThera, Comments: medical advisory board; I have received no fees to date but will.

\section{REFERENCES}

1. Rykken JB, Diehn FE, Hunt CH, et al. Rim and flame signs: postgadolinium MRI findings specific for non-CNS intramedullary spinal cord metastases. AJNR Am J Neuroradiol 2013;34:908-15

2. Rykken JB, Diehn FE, Hunt $\mathrm{CH}$, et al. Intramedullary spinal cord metastases: MRI and relevant clinical features from a 13-year institutional case series. AJNR Am J Neuroradiol 2013;34:2043-49

3. Mostardi PM, Diehn FE, Rykken JB, et al. Intramedullary spinal cord metastases: visibility on PET and correlation with MRI features. AJNR Am J Neuroradiol 2014;35:196-201

4. Sung WS, Sung MJ, Chan JH, et al. Intramedullary spinal cord metastases: a 20-year institutional experience with a comprehensive literature review. World Neurosurg 2013;79:576-84

5. Wilson DA, Fusco DJ, Uschold TD, et al. Survival and functional outcome after surgical resection of intramedullary spinal cord metastases. World Neurosurg 2012;77:370-74

6. Connolly ES Jr, Winfree CJ, McCormick PC, et al. Intramedullary spinal cord metastasis: report of three cases and review of the literature. Surg Neurol 1996;46:329-37; discussion 337-38

7. Lee SS, Kim MK, Sym SJ, et al. Intramedullary spinal cord metastases: a single-institution experience. J Neurooncol 2007;84: $85-89$ 